\title{
A dynamical systems model for poly-cyclic foliar forest pathogens
}

\author{
$\begin{array}{lll}\text { G. Wake }^{1} & \text { N. Williams } & \text { T. Pleasants } \\ & \end{array}$
}

Received 14 November 2017; revised 29 January 2018

\begin{abstract}
A simple systems model is proposed to understand and quantify the onset and epidemiology of red needle cast in radiata pine. This disease is impacting much of the New Zealand forestry estate being driven through the production of self-replicating spores which are dispersed with water. The model is at present deterministic, not spatially or age-structured, nor dependent on environmental or seasonal effects. This model shows the clear existence of calculable thresholds for disease proliferation and elimination, showing it has captured the essential components of the biological mechanisms. It is to be used to identify thresholds for infection to spread or retract. Further it will provide a base model from which we can fit and then predict experimental outcomes.
\end{abstract}

Subject class: $92 \mathrm{Bo} 5$

DoI:10.21914/anziamj.v59i0.12625, (c) Austral. Mathematical Soc. 2018. Published April 5, 2018, as part of the Proceedings of the 13th Biennial Engineering Mathematics and Applications Conference. ISSN 1445-8810. (Print two pages per sheet of paper.) Copies of this article must not be made otherwise available on the internet; instead link directly to the DOI for this article. 
Keywords: Tree disease, epidemiology, systems model

\section{Contents}

1 Introduction

2 Materials and Methods

3 Results

4 Discussion

C11

References

C12

\section{Introduction}

Standard epidemiological models are central to describing and understanding the dynamics of infection, inoculum build up and symptom expression of plant disease. These have been applied to a range of plant disease epidemics with the establishment of the model providing a framework for epidemiological studies $[1,6,7,10]$. Each of these studies gives rise to different functional expressions in the systems model. The temporal dynamic relationship between the availability of susceptible plant material (S), the rate at which it becomes infected and displays disease (I) after the introduction of a pathogen $(\mathrm{P})$ is impacted by numerous genetic and environmental conditions and the accompanying biological state of the both the pathogen and host material. These dynamic relationships can be impacted by changes to a range of host, pathogen or environmental factors such as humidity, rainfall and temperature often targeted by integrated pest management approaches in managed forest systems. For example, selection of resistant germplasm, manipulation of environmental conditions through site selection or silviculture and the 
implementation of chemical or biological controls can each target components of the interaction between host and pathogen integral to sustained disease management. However, forest pathology studies rarely integrate these studies in dynamic models to demonstrate the impact of these systems model strategies on the pathogen population and long-term outcomes for disease management. In this paper, a prototype simple mathematical model is presented to describe the dynamics of red needle cast (RNC) in New Zealand's Pinus radiata plantations. This disease is caused by the foliar pathogen Phytophthora pluvialis, and has been observed to have a polycyclic disease cycle driven by the reproduction of spores and further infection of plant material under environmental conditions favourable to disease [2]. It is prevalent in pine trees and manifests itself within pine tree needles which form lesions then turn yellow and red-brown, before being cast from the tree. It is transmitted by these fungal spores released into water films on the needle surfaces under conditions of high humidity and persistent rain.

Much data has been collated showing RNC is sensitive to environmental conditions [3], host susceptibility [4], and chemical control [11, 12] affording considerable opportunity for disease management. This has yet to be used in conjunction with a dynamic systems model in which time is the main independent variable, nor has it been collected to enable such a model to be audited and parameterised. This paper addresses this by proposing a simple biological- and epidemiological-based model integrating the potential impacts of control measures to provide a framework for improving decision support and research of the complexities of the disease. Establishment of the model provides a structural clarity for the collection and curation of field and laboratory data pertaining to the disease and infection. Once suitable timeseries data is available, the model will be empirically parameterised and tested against new data. Then it will be used to underpin control measures and predictions as to its spread and impact on the growth of healthy and infected pine trees. This will help with the timber production in New Zealand and elsewhere in response to this and other foliar diseases in forestry plantations. 
New born needles

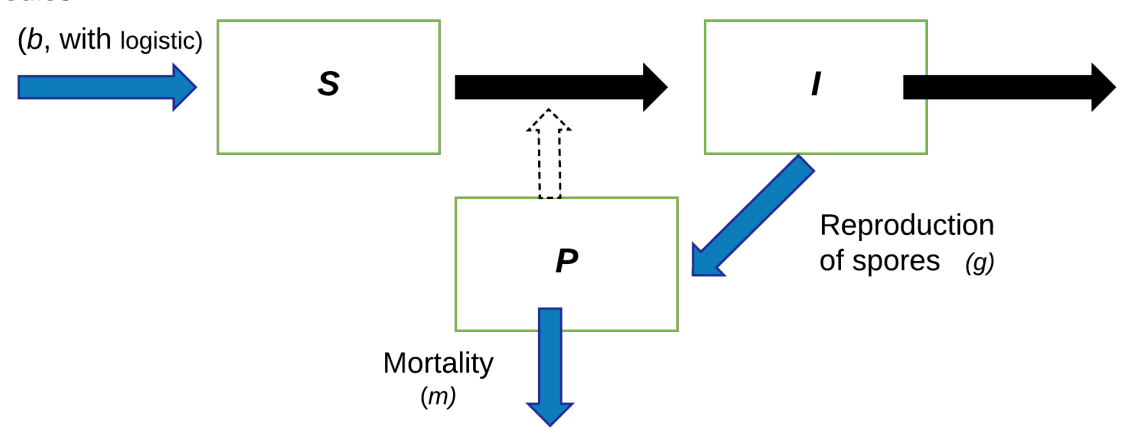

Figure 1: Schematic diagram showing the flow between the three key model compartments, susceptible-healthy pine needles (S), infected needles (I) and the inoculum load of the pathogen $(\mathrm{P})$ along withe the key model parameters $\mathrm{b}, \mathrm{k}, \mathrm{g}$ and $\mathrm{m}$.

\section{Materials and Methods}

The model is motivated by the similar model created to address similar epidemiological inter-actions such as that of the PSA bacterial epidemics encountered recently in the commercial production of the Kiwifruit [9, 8]. That model was developed to help understand the dynamics of infection and use of bio-controls, called elicitors, to induced plant resistance. The diagram in Figure 1 shows how the essential compartments (and their interactions) occur from a given initial condition in the pine needle model shown in the Figure 1. Three compartments are shown: susceptible needles (S), Infected pine needles (I), and spore density (P). Because of the considerable difficulty of accumulating sufficient data for this purpose, the first two compartments are taken as proportions, and so $\mathrm{S}+\mathrm{I}=1$.

At its fundamental level, the key parameters of RNC infection and the resulting 
disease expression can be explained by two compartmented variables of needle health and spore density which are underpinned by the physiology of the pathogen and its host within their mutual environment. In developing the model these were treated as unstructured variables and did not take into consideration factors of host age, size or spatial relationship to enable application within experimental and field based contests going forward. The model states that pine needles are in one of two states: susceptible, but not yet infected (i.e. healthy) (S) and infected (I), measured as proportions, so at the time when healthy plants $(S)$ encounter pathogen spores $(t=0)$, a proportion of the plant population will become infected (I) at a rate given as the product of $(\mathrm{S})$ and $(\mathrm{P})$ assuming mass-action transmission of the disease and with transmission coefficient ( $\mathrm{k}$ ). Accordingly, as noted above, $\mathrm{I}=1-\mathrm{S}$. These infected needles then give the potential of the pathogen to reproduce further spores governed by the rate of reproduction $(\mathrm{g})$. The build-up of the pathogen spore inoculum (P) over time feeds back into the dynamic of infection but is tempered by the mortality rate of spores $(m)$ and emergence of new needles available for infection (b) and the loss of infected needles from the system. As with all classic epidemialogical models, red needle cast was found to conform to a logistic growth model constrained by the availability of susceptible plant material and driven by the build-up of spore from infected material. The model's equations for plant infection are expressed by the coupled mass-balance two-dynamical system as follows:

Logistic growth with mass-action transmission

$$
\dot{S} \equiv \frac{\mathrm{d} S}{\mathrm{dt}}=\mathrm{bS}(1-\mathrm{S})-\mathrm{kSP} ;
$$

and initial condition taken as

$$
\mathrm{S}(\mathrm{t}=0)=\mathrm{So}=1 .
$$

Logistic growth affected by infection and natural death of spores

$$
\dot{\mathrm{P}} \equiv \frac{\mathrm{dP}}{\mathrm{dt}}=\mathrm{gIP}\left(1-\frac{\mathrm{P}}{\mathrm{K}_{1}}\right)-\mathrm{mP} ;
$$


with initial condition

$$
\mathrm{P}(\mathrm{t}=0)=\mathrm{Po} \quad \text { (to be varied }) .
$$

As an auxiliary equation the third compartment (I) which of course satisfies matching growth and death terms

$$
\dot{\mathrm{I}}=\frac{\mathrm{dI}}{\mathrm{dt}}=\mathrm{k}(1-\mathrm{I}) \mathrm{P}-\mathrm{b}(1+\mathrm{I}) \mathrm{I} ;
$$

with initial condition $\mathrm{I}(0)=0$.

This ensures that the proportions satisfy $S+I=1$. The last equation in (3) is not needed to be solved for I explicitly but it provides future scope for exploring the dynamics of inoculum build-up and disease transmission. The assumption of logistic growth of healthy needles and removal of infected needles is to be tested by data eventually.

The model is therefore articulated with just two differential equations of the change in needle health over time (equation (1)) with mass-action kinetics for the infection, and the change in pathogen load over time (equation (2)). The occurrence of the "I" term in the growth rate of the spores indicates that the degree of infection assists the production of more spores. Here we take $k$ as the transmission parameter with units $[k]=$ Days $^{-1}$ and $m$ is the percapita death-rate of spores per needle $[\mathrm{m}]=$ Days $^{-1}$ and $\mathrm{Po}_{\mathrm{o}}$ is the initial spore concentration (Table 1). For equation (2), $\mathrm{I}=1-\mathrm{S}$ removing the need for a third equation. Biological insight suggests there should be a delay effect in"I" in equation (2), but this is simply signalled and will not be explored further here. For the purpose of establishing the theoretical model, the unknown parameters $\left(\mathrm{b}, \mathrm{g}, \mathrm{m}, \mathrm{k}, \mathrm{K}_{1}, \mathrm{Po}\right)$, were parameterised based on laboratory observations of the pathogen, latency of infection and symptom development observed through direct inoculation studies and are provided in Table 1 using estimates based on preliminary analysis. The two significant ones $(m, k)$ are then varied to determine an outcome diagram given in Figure 3 later, by considering the occurrence and swaps in stability of the steady-states of the system described by these equations in (1) and (2). 
Table 1: Dimensions of parameters and state variables applied in model development

\begin{tabular}{|c|c|c|}
\hline Description \& Symbol & Value & Units \\
\hline Per-capita rate of needle emergence $b$ & 0.16 & Days $^{-1}$ \\
\hline Per-capita birth-rate of spores $g$ & 0.68 & Days $^{-1}$ \\
\hline Per-capita death-rate of spores $m$ (control variable) & 0.01 & Days $^{-1}$ \\
\hline Carrying capacity of spores for each needle $\mathrm{K}_{1}$ & 50 & Number \\
\hline Initial spore count Po (control variable) & 10 & Number \\
\hline Infectivity of each spore $k$ (control variable) & 0.01 & Days $^{-1}$ \\
\hline
\end{tabular}

\section{Results}

The computed solutions were produced in MatLab and $\mathbf{R}$ using computerbased algorithms (such as ode45) and plotted using the parameter values (Table 1). An illustrative plot only is shown in Figure 2. This illustrates the characteristics of the two-compartments $\mathbf{S}$ and $\mathbf{P}$ for equations (1) and (2) Exploration of this model for which the proportion of healthy needles $S$ ranges between 0 and 1 and the concentration of spores $P$ is greater or equal to 0 identified five steady states, all of which appear feasible given the expression of the disease and underlying pathology principles. Depending on the parameters, there are abrupt changes between steady-states as parameters are changed with swaps in the stability. We note that although the system is normally operating temporally, far from equilibrium, the steady states drive the temporal behaviour. Of course these steady-states are evaluated by setting the righthand sides of equations (1) and (2) to zero. Which gives rise to the output diagram in Figure 3.

The first two states are the absence of either the host plant $(S=0$, so $\mathrm{P}=0)$ or pathogen $(S=1, P=0)$ from the system and thus a lack of interaction between the two. Both describe the primary objectives of biosecurity through the exclusion of the pathogen by quarantine or destruction of host material during eradication attempts. Checking the Jacobian matrix at the first 

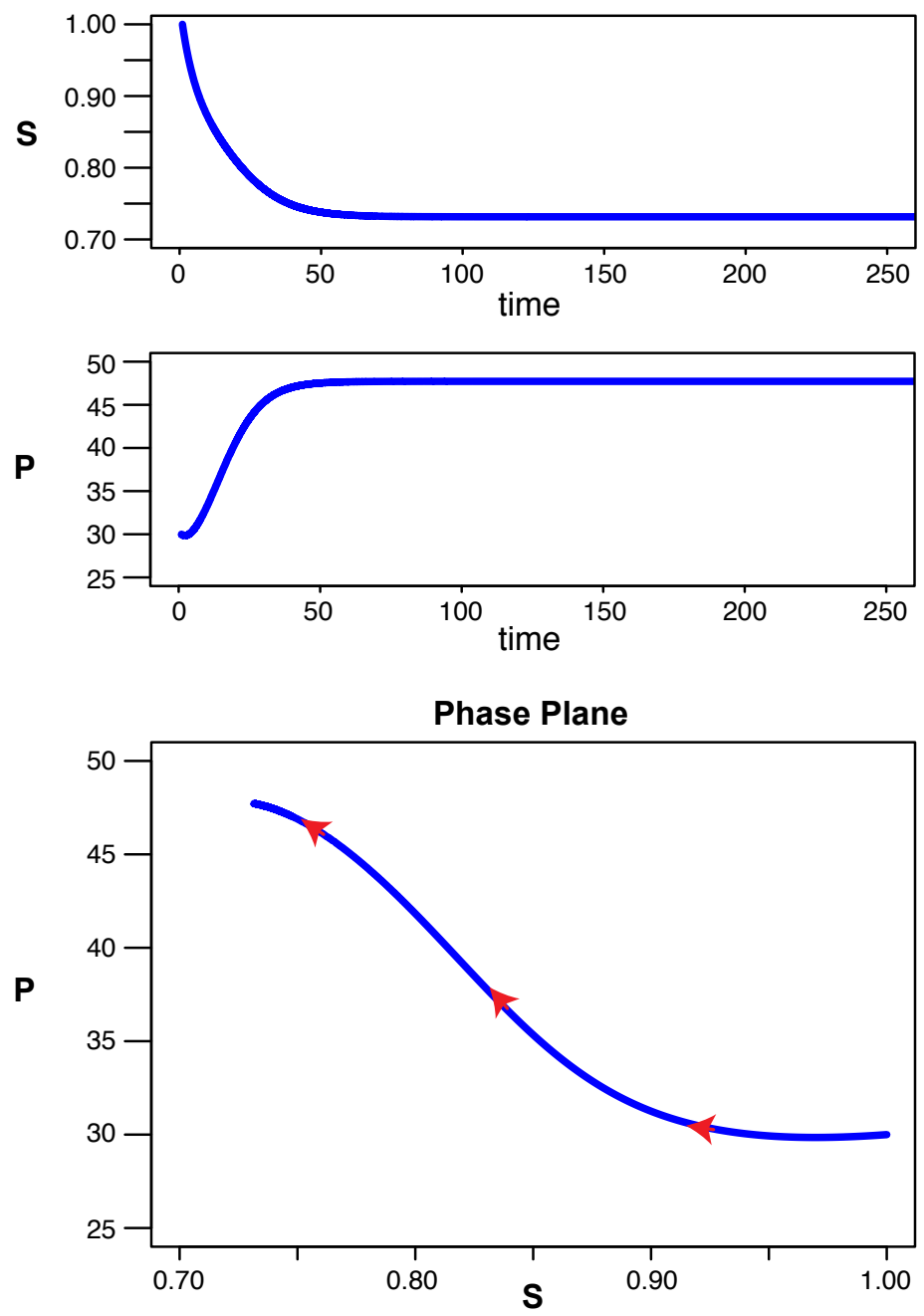

Figure 2: Typical time-evolutions and corresponding phase-plane for the RNC dynamic model. Here the initial condition has the tree initially being perfectly healthy $(S=1)$, receiving an initial invasive inoculation $P o=30$ units. The outcome, in this case, is that the disease partially overcomes the plant, with the spore count ultimately approaching its carrying capacity. The arrows indicate the time-elapsed from initially $(S=1)$ at $t=0$ initially through to much later times. 
(extinction) steady-state reveals it is always unstable (a saddle). So it is therefore infeasible, as expected.

The third steady state describes uncontrolled disease in the absence of management intervention region: [3.1] in Figure 3. In this scenario $S=0$ and the reproductive rate of spores is greater than their natural mortality so the long-term outcome is fully-diseased. In the absence of active control this dynamic will be subject to constraints of host susceptibility and environment. The stability of this state is subject to these controls and, when stable, has a computable basin of attraction when Po is large enough.

The next two steady states are the only two that co-exist and describe partially diseased population for which $0<S<1$. Depending on the values $(\mathrm{m}, \mathrm{k})$ one of these is inherently unstable (so does not appear in Figure 2) and the stability of the other varies in response to the dynamics of the model constraints as described above regions $[3.2,3.3]$ in Figure 3.

A fourth applicable state exists for all values of $k$ for which the rate of spore mortality $(\mathrm{m})$ exceeds their regeneration potential $(\mathrm{g})$ see region [3.4] in Figure 3. This describes the scenario for an incompatible interaction of host-pathogen and/or environment, low susceptibility disease and universal disease control. Under such conditions, the disease will not establish itself. Hence for practical applications, $k$ only matters when $m<g$. These stable and unstable steady states provide useful insights for the investigation of RNC disease dynamics.

In the outcome diagram below (Figure 3) the two parameters most amenable to control, the disease transmission coefficient $k$ and the spore death rate $m$. are plotted showing the sharp demarcation between different outcomes.

For the informed values of the parameters in Table 1, the scenario envisaged sits in Region [3.1]. This, when and if verified, will be very significant as then the value of the initial spore concentration determines the long-term outcome, suggesting that this is a third important matter for determining control strategies. 


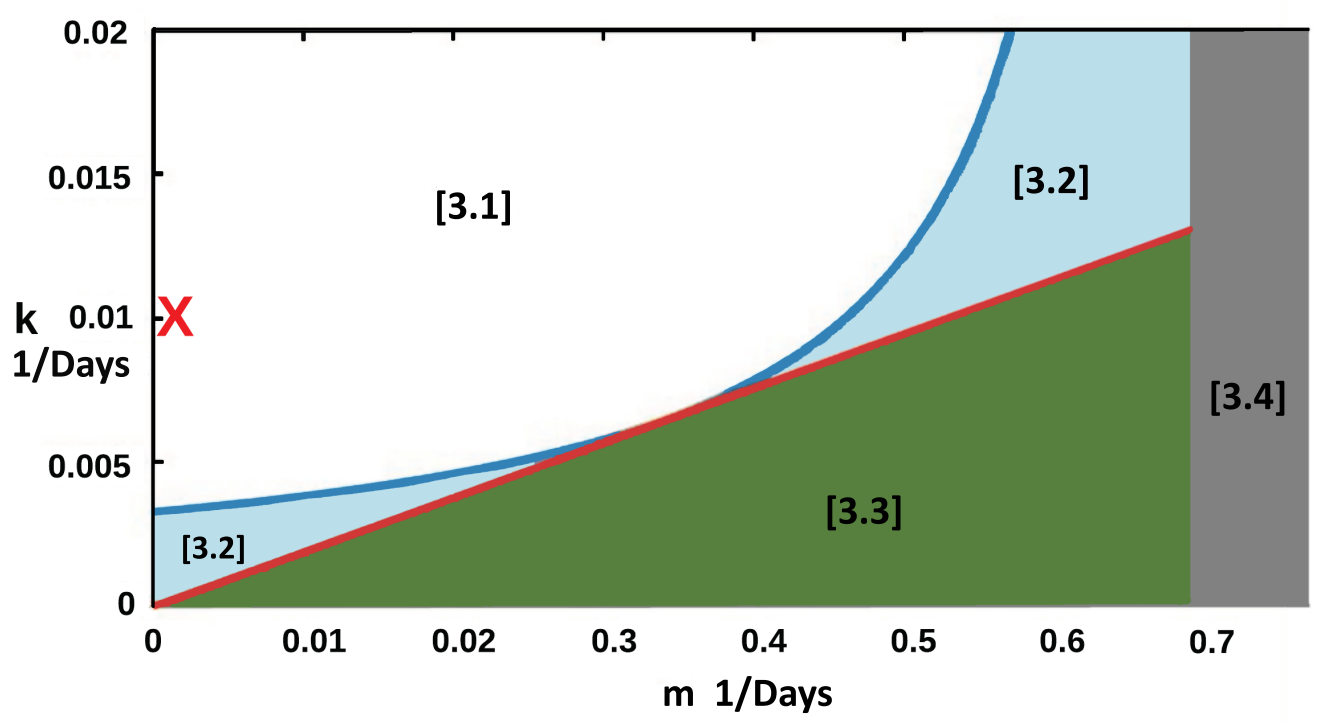

Figure 3: Scenarios of RNC disease is explained by four situations. In region [3.1] of the parameter-space there are values for $k$ and $m$ which result in uncontrolled disease; region [3.2] has unstable states in which tipping points exist that will push the disease either into a healthy or diseased state and region [3.3] conditions under which disease will be controlled. A fourth situation [3.4] exists in which $\mathrm{m}>\mathrm{g}$ for all $\mathrm{k}$, such that $P$ pluvialis mortality is greater than the birth rate of spores, leading to a finally healthy plant. The lines of demarcation are obtained analytically, with the curved line being a rectangular hyperbola $k=b /\left(K_{1}(1-m / g)\right)$. The cross $X$ indicates the outcome with the values of $k, m$ in Table 1 . 


\section{Discussion}

The management of plant disease in forest systems involves assessment of the dynamic relationship at play between host, pathogen and environment over time. Epidemiological models of disease dynamics serve as key decision support tools to facilitate management and better understand the drivers of disease epidemics. By articulating disease dynamics with respect to the epidemiological drivers of the disease, complex interactions between the pathogen, host, environment and the impact of management intervention can be better understood and quantified. This will in turn signal likely control strategies. The framework going forward will be to incorporate environmental effects, spatial structure, etc., while keeping the essential dynamics described here. Code for this analysis is available [5]. Sensitivity analysis is needed as well to elucidate the defining nature of the four regions shown in Figure 3.

In this study, we discuss the development of a prototype mathematical model to describe the dynamics of red needle cast in New Zealand's Pinus radiata plantations. This disease is now well established throughout much of New Zealand's forested areas [3] which of considerable significance for their productivity and New Zealand's wood production. The current model described here offers the potential to quantitatively estimate the impact of subtle changes in parameters impacting the build-up of inoculum and host susceptibility over time. While simple and generic in its nature, the model provides a framework to better understand the dynamics of RNC development in both naturally infected plants and controlled laboratory, growth-room and field based studies of the disease. In ongoing work, this prototype SI model will be refined to better predict the core parameters driving disease dynamics with focus on elucidating the key parameters of $k, g$ and $m$ and how these many be exploited by disease management. The inclusion of stochastic elements is yet to be explored but will further aid the application of the model in the field. Extensions to the model with field data will provide a powerful tool for the construction of environmental and geospatial disease risk models to inform management. Furthermore, this model provides a framework for 
better understanding the direct interactions between the pathogen and host material displaying a range of susceptibilities to disease. The flexibility of this simplified model thus provides a strong framework on which to collate, articulate and target $\mathrm{RNC}$ research going forward.

Acknowledgements Our thanks are extended to Faheem Zaidi, PhD candidate, Massey University and Rebecca Turner, $\mathrm{PhD}$ candidate University of Auckland for their assistance in the model computations and producing the figures for publication. We thank Lindsay Bulman, Natalie Graham, Ralf Gommers and Heidi Dungey of (Scion Research) for their contribution to early discussions during model development. This work was funded by the New Zealand Ministry of Business, Innovation and Employment, The New Zealand Forest Grower's Levy Trust and Ministry for Primary Industries under the Healthy Trees, Healthy Future program (MBIE C04X1305). Thanks are also extended to the referees for their detailed comments which are incorporated and have greatly improved the manuscript.

\section{References}

[1] C Lee Campbell, Laurence V Madden, et al. Introduction to plant disease epidemiology. John Wiley \& Sons., 1990. C2

[2] Margaret Anne Dick, Nari Michelle Williams, Martin Karl-Friedrich Bader, Judy Frances Gardner, and Lindsay Stuart Bulman.

Pathogenicity of phytophthora pluvialis to pinus radiata and its relation with red needle cast disease in new zealand. New Zealand Journal of Forestry Science, 44(1):6, 2014. C3

[3] Heidi S Dungey, Nari M Williams, Charlie B Low, and Graham T Stovold. First evidence of genetic-based tolerance to red needle cast caused by phytophthora pluvialis in radiata pine. New Zealand Journal of Forestry Science, 44(1):31, 2014. C3, C11 
[4] RJ Ganley, NM Williams, CA Rolando, IA Hood, HS Dungey, PN Beets, LS Bulman, et al. Management of red needle cast, caused by phytophthora pluvialis, a new disease of radiata pine in new zealand. $N$. Z. Plant Protect.-SE, 67:48-53, 2014. C3

[5] Wake GC. Private communication, 2017. C11

[6] Christopher A Gilligan. An epidemiological framework for disease management. Advances in botanical research, 38:1-64, 2002. C2

[7] MJ Jeger. Asymptotic behaviour and threshold criteria in model plant disease epidemics. Plant Pathology, 35(3):355-361, 1986. C2

[8] Nurul S Abdul Latif, Graeme C Wake, Tony Reglinski, and Philip AG Elmer. Modelling induced resistance to plant diseases. Journal of theoretical biology, 347:144-150, 2014. C4

[9] Nurul S Abdul Latif, Graeme C Wake, Tony Reglinski, Philip AG Elmer, and Joseph T Taylor. Modeling induced resistance to plant disease using a dynamical systems approach. Frontiers in plant science, 4, 2013. C4

[10] Laurence V Madden, Gareth Hughes, Frank Bosch, et al. The study of plant disease epidemics. American Phytopathological Society (APS Press), 2007. C2

[11] CA Rolando, MA Dick, J Gardner, M Bader, NM Williams, et al. Chemical control of two phytophthora species infecting the canopy of monterey pine (pinus radiata). Forest Pathology, 47(3), 2017. C3

[12] Carol Rolando, Robyn Gaskin, David Horgan, Nari Williams, and Martin KF Bader. The use of adjuvants to improve uptake of phosphorous acid applied to pinus radiata needles for control of foliar phytophthora diseases. New Zealand Journal of Forestry Science, 44(1):8, 2014. C3 


\section{Author addresses}

1. G. Wake, Institute of Natural and Mathematical Sciences and Al Rae Centre for Genetics and Animal Breeding, Massey University, New Zealand New Zealand.

mailto:g.c.wake@massey.ac.nz

2. N. Williams, Scion, Forest Protection, Scion, Rotorua, New ZEALAND.

mailto:nari.williams@scionresearch.co.nz

3. T. Pleasants, Al Rae Centre for Genetics and Animal Breeding, Massey University, Hamilton,New ZeALAND.

mailto:tony@pleasantsanalytics.co.nz 